Bangladesh J. Plant Taxon. 19(1): 63-78, 2012 (June)

(C) 2012 Bangladesh Association of Plant Taxonomists

\title{
A SYNOPTICAL ACCOUNT OF THE STERCULIACEAE IN BANGLADESH
}

\author{
M. Oliur Rahman ${ }^{1}$, Md. Abul Hassan, Md. Manzurul Kadir Mia ${ }^{2}$ \\ ANd AHMed Mozaharul Huq ${ }^{3}$ \\ Department of Botany, University of Dhaka, Dhaka 1000, Bangladesh
}

Keywords: Taxonomy; Sterculiaceae; Nomenclature; Distribution; Bangladesh.

\begin{abstract}
Taxonomy, updated nomenclature and occurrence of the species belonging to the family Sterculiaceae in Bangladesh have been presented. Detailed herbarium study at Royal Botanic Gardens, Kew (K), Royal Botanic Garden, Edinburgh (E), British Museum (BM), Bangladesh National Herbarium (DACB) and Dhaka University Salar Khan Herbarium (DUSH) has revealed the occurrence of 32 species under 15 genera of the Sterculiaceae in Bangladesh. The correct name, important synonym(s), salient diagnostic characteristics, specimens examined and distributional notes have been provided for each species. Dichotomous bracketed keys have also been presented for identification of genera and species.
\end{abstract}

\section{Introduction}

The Sterculiaceae is a family of tropical and sub-tropical plants, comprising nearly 70 genera and 1,500 species (Cronquist, 1981). They are characterized by the presence of stellate hairs, bilocular anthers, 10 to numerous stamens in two or more whorls, mostly connate by their filaments, superior ovary, anatropous ovules and axile placentation. The family consists of softwooded trees and shrubs, and a few herbaceous and climbing species. Many species growing in rain forests are remarkable for their development of plank buttresses. Systematically it is placed in the Malvales by Engler and Prantle (1896), and shows many features in common with the other families of that group, namely Tiliaceae, Elaeocarpaceae, Bombacaceae and Malvaceae. Evidence from floral anatomy and embryology suggests that the Sterculiaceae is the most primitive in the Malvales (Brizicky, 1966). Hooker (1874) recognized six tribes of the Sterculiaceae, viz., Sterculieae, Helictereae, Eriolaeneae, Dombeyeae, Hermannieae and Buettnerieae (Byttnerieae). Based on floral anatomy and embryology the tribe Sterculieae is regarded as the most primitive because of the presence of pentacyclic flowers, numerous stamens, apocarpous gynoecia, and many ovulate locules, while the Hermannieae is the most advanced tribe as it possesses tetracyclic flowers, a reduced number of stamens and ovules, and a tendency of perigyny. In respect to perianth structure, the Helictereae and Byttnerieae appear to be the most highly specialized tribes of Sterculiaceae (Brizicky, 1966).

Several taxonomic treatments on the Sterculiaceae have been made based on morphology (Robyns and Cautrecasas, 1964; Hsiang-Hao, 1984; Malick, 1993; Verdcourt, 1995; Phengklai, 2001). Hooker (1874) worked out the Sterculiaceous taxa of British India, while Prain (1903) dealt with the species of the then Bengal documenting 20 species and 9 species, respectively from the area of present Bangladesh. Despite Khan (1972-1989), and Khan and Rahman (1989-2002) published the Flora of Bangladesh in different fascicles covering several angiospermic families, the Sterculiaceae was not included in those treatments. Ahmed et al. (2009) listed 25 species of

\footnotetext{
${ }^{1}$ Corresponding author. Email: dr_oliur@yahoo.com

${ }^{2}$ Former Principal Scientific Officer, Bangladesh National Herbarium, Mirpur 1, Dhaka 1216, Bangladesh.

${ }^{3}$ Former Consultant-Taxonomist, University of Illinois at Chicago, USA.
} 
Sterculiaceae under 13 genera from Bangladesh. Very recently Mia et al. (2011) reported three species of Sterculiaceae, namely Guazuma ulmifolia Lam., Helicteres viscida Bl. and Sterculia urens Roxb. as new distributional records for Bangladesh. However, no detailed study was carried out on this family so far. Moreover, plants of Bangladesh belonging to this family deposited in different foreign herbaria, particularly Royal Botanic Gardens, Kew (K), Royal Botanic Garden, Edinburgh (E) and British Museum (BM) have never been investigated earlier. The present study aimed at studying all species of Sterculiaceae of Bangladesh deposited in the herbaria both at home and abroad, and documenting and detailing all members of the family occurring in Bangladesh along with their updated nomenclatural and distributional notes.

\section{Materials and Methods}

The present study is based on both literature survey and herbarium studies. Plants deposited at K, E, BM, Bangladesh National Herbarium (DACB) and Dhaka University Salar Khan herbarium (DUSH) have been examined critically. Dichotomous artificial keys have been provided for identification of genera and species. Nomenclature has been updated for each species along with their important synonyms following Verdcourt (1995), Phengklai (2001) and Ahmed et al. (2009). Salient diagnostic characters, specimens examined and distributional notes have also been furnished under each species.

\section{Results}

The present study revealed the occurrence of 32 species under 15 genera of the family Sterculiaceae in Bangladesh. A dichotomous artificial key to genera of the Sterculiaceae is given below.

\section{Key to the genera:}

1. Leaves simple.

- $\quad$ Leaves compound.

Sterculia

2. Petals present; flowers bisexual or polygamous; herbs, shrubs or trees.

- $\quad$ Petals absent; flowers unisexual or polygamous; trees or shrub

3. Ovary stipitate.

- $\quad$ Ovary sessile.

4. Anther loculi parallel, linear; seeds winged.

- $\quad$ Anther loculi divaricate; seeds not winged.

Pterospermum

5. Mature carpels inflated; flowers in a large terminal panicle.

- Mature carpels not inflated, but often spirally twisted; flowers solitary or fasciculated.

6. Capsules 5-winged; staminodes emarginate.

Abroma

Capsules not winged; staminodes acute.

7. Fertile stamens 5; capsules covered with stiff, long spines or prickles. 
8. Petals with hooded claw. Capsules tuberculate, muricate with soft plumose bristles.

- $\quad$ Petals without hooded claw. 9

9. Petals mostly persistent and often enlarged after flowering. $\quad \mathbf{1 0}$

- $\quad$ Petals deciduous or very inconspicuous after flowering. $\quad \mathbf{1 1}$

10. Annual herbs; flowers usually solitary. Pentapetes

- $\quad$ Trees and shrubs; flowers rarely solitary. Dombeya

11. Ovary 5-locular; styles $5 . \quad$ Melochia

- $\quad$ Ovary 1-locular; style $1 . \quad$ Waltheria

12. Fruiting carpels strongly keeled, indehiscent, 1- seeded. Heritiera

- $\quad$ Fruiting carpels slightly keeled, 1- many seeded. $\quad 13$

13. Anthers regularly arranged in one or more whorls. Seeds several, Pterygota attached along the entire margin of open carpels, distinctly winged.

- Anthers irregularly crowded in a cluster at the apex of $\mathbf{1 4}$ androgynophore.

14. Leaves palmately lobed; fruits membranous; seeds borne on margin of carpels.

Firmiana Leaves not palmately lobed; fruits leafy; seeds not borne on margin of carpels.

Scaphium

ABROMA Jacq., Hort. Vindob. 3: t. 1 (1776); Benth. \& Hook. f., Gen. P1. 1: 224 (1862).

Type: Theobroma augusta L. [Abroma augusta (L.) L. f.]

Abroma augusta (L.) L. f., Suppl.: 341 (1781) ('Ambroma'); Mast. in Hook. f., Fl. Brit. Ind. 1: 375 (1874); Prain, Beng. P1. 1: 278 (1903); Ridl., Fl. Mal. Pen. 1: 286 (1922); Craib, Fl. Siam. Enum. 1: 179 (1925); Malick in Sharma \& Sanjappa (eds), Fl. India 3: 409 (1993). Theobroma augusta L., Syst. Veg. ed. 13: 580 (1774).

A shrub or small tree. Leaves cordate, repand-denticulate, with acuminate to cuspidate apex and cordate base, base 3-7 nerved. Flowers dark red. Fruit a capsule, conical, winged. Flowering and fruiting: June-December.

Specimens examined: Chittagong: s. loc. \& dies, J.M. Cowan 179 (E). Rangapani, 30.10.1978, Huq, Rahman \& Mia, H. 4005 (DACB). Dhaka: Dhaka University Botanic Garden, 13.9.1949, s. n. (DUSH); Ramna, 15.8.1940, Atul, s. n. (DUSH); Bangladesh National Herbarium Compound, Mirpur, 20.1.2004, Rezia Khatun 4644 (DACB). Faridpur: Gualondo ghat, 1.7.1973, A.M. Huq 982 (DACB). Manikganj: Taraghat village, 6.6.1978, Soejarto \& Rahman 4976 (K). Rangamati: Bilaichari, Farua Reserve forest, 15.10.2008, S.N. Uddin 3056 (DACB). Sylhet: Wallich no. 1142 (K).

Distribution: Australia, Bhutan, China, India, Indonesia, Malaysia, Nepal, Pacific islands, Philippines, Thailand and Vietnam. 
BYTTNERIA Loeft., Iter. Hisp. : 313 (1758); Buettneria L, Syst. Veg. ed. 13: 197 (1774); Benth. \& Hook. f., Gen. P1. 1: 225 (1867).

Type : Byttneria scabra Loeft. ex L.

\section{Key to the species:}

1. Leaves entire, glabrescent above and puberulous on nerves

B. aspera beneath; inflorescence minutely puberulous; flower buds ovoid.

- $\quad$ Leaves finely serrate-dentate, stellate-pubescent on both

B. pilosa surfaces; inflorescence densely stellate-pubescent; flower buds conical.

Byttneria aspera Colebr. in Roxb., Fl. Ind. ed. Carey 2: 383 (1824); Mast. in Hook. f., Fl. Brit. Ind. 1: 377 (1874); Prain, Beng. P1. 1: 279 (1903); Kanjilal et al., Fl. Assam 1: 160 (1934). Byttneria grandifolia DC., Prod. 1: 486 (1824); Malick in Sharma \& Sanjappa (eds), Fl. India 3: 410 (1993). Byttneria integrifolia Lace, Kew Bull. 1915: 396 (1915); Craib, Fl. Siam. Enum. 1: 181 (1925). Byttneria siamensis Craib, Kew Bull. 1920: 300 (1920).

A woody climber or scandent shrub. Leaves cordate with cuspidate apex and cordate base, 5-7 nerved at the base. Flowers greenish, minute, $4-5 \mathrm{~mm}$ across. Fruit a capsule, globose, sparsely stellate hairy, armed. Flowering and fruiting: May-December.

Specimens examined: Chittagong: South-west of Hazarikhil, 1.11.1978, Huq, Rahman \& Mia, H 4115 (DACB); Chunati Wildlife Sanctuary, 30.12.1989, Khan \& Huq, K 8188 (DACB). Sylhet: Wallich no. 1144, 2 (BM).

Distribution: Bhutan, Cambodia, China, India, Laos, Nepal, Thailand and Vietnam.

Byttneria pilosa Roxb., Fl. Ind. 2: 681 (1832); Mast. in Hook. f., Fl. Brit. Ind. 1: 377 (1874); Kurz, Fl. Burm. 1: 151 (1877); Craib, Fl. Siam. Enum. 1: 181 (1925) 'Buettneria’ Prain, Beng. P1. 1: 279 (1903). Malick in Sharma \& Sanjappa (eds), Fl. India 3: 414 (1993). Buettneria elegans Ridl., J. Straits Roy. Asiat. Soc. 57: 25 (1911); Craib, Fl. Siam. Enum. 1: 181 (1925).

A large scandent shrub or woody climber with grooved hispid branchlets. Leaves suborbicular, palmately lobed with acute or cuspidate apex and cordate base, stellate pilose on both surfaces. Flowers pale yellow, 4-6 mm across. Fruit a globose capsule, with black, hairy spiny tubercules. Flowering and fruiting: September-February.

Specimens examined: Bandarban: s. loc., 29.2.1868, C.B. Clarke 6591 (K); Ruma Bazar, 28.10.1984, Khan, Huq, Rahman and Mia, K 6723 (DACB). Chittagong: Jaldi Range, 13.10.1920, J.M. Cowan 112 (E); Jaldi Range, 10.12.1920, J.M. Cowan 1672 (E); Garjania, J.M. Cowan 453 (E), Chittagong, 5.1.1851, J.D. Hooker 365 (K); Chittagong, 28.11.1850, J.D. Hooker s.n. (K); Chittagong, Barharcherry, 4.2.1873. C.B. Clarke 19584 A (BM); Chittagong, J.D. Hooker \& T. Thamson s.n. (BM); Sitakund, 12.3.1968, M.A.R. Laskar 88 (DUSH); Hazarikhill, Sitakund, 24.1.1968, M. Akram Hossain 96 (DUSH). Chittagong Hill Tracts: s. loc., D. King's Coll. 57 (BM); Near Sangunakropara, 17.1.1965, M.S. Khan 915 (DUSH); Kendachari, 12.11.1968, Alo Rani 78 (DUSH). Cox's Bazar: Jadi Pahar, 28.12.1957, M.S. Khan 289 (DUSH). Maulvi Bazar: Lowachara forest, 19.1.1963, M.S. Khan 467 (DUSH). Rangamati: Kaptai, Tinconia road, 25.9.1934, Parkinson 4298 (K). Sherpur: Gajni forest area, 27.4.1986, Huq \& Mia, H 7692 (DACB). Sylhet: s. loc. \& dies, Wallich 1145 (K); Sylhet 9.10.1872, C.B. Clarke 17422A (BM).

Distribution: China, India, Indonesia, Laos, Malaysia, Myanmar, Thailand and Vietnam. 
DOMBEYA Cav., Diss. 121: t. 38, 41 (1787).

\section{Key to species:}

1. Peduncle more than $20 \mathrm{~cm}$ long; sepals linear-oblong;

D. wallichii stigma exserted.

- $\quad$ Peduncle up to $8 \mathrm{~cm}$ long; sepals lanceolate; stigma

D. mastersii included.

Dombeya mastersii Hook. f., Bot. Mag. 93: t. 5639 (1867). Dombeya angulata auct. non Cav. (1867).

A stellate tomentose shrub. Leaves broadly ovate, entire or occasionally obscurely 3-lobed, deeply cordate at the base, acute to acuminate at the apex. Flowers white or pinkish-white. Stigma included. Fruit a capsule, oblong, villous. Planted in the gardens. Flowering and fruiting: December-April.

Specimens examined: Dhaka: Dhaka University Botanic Garden, 15.3.1943, s.n. (DUSH); Ramna Park, 31.12.1962, S.K. Dutta 61 (DUSH); Balda Garden, 20.1.1966, S.K. Dutta 76 (DUSH); Ramna park, 19.2.1980, Momtaz Begum 196 (DACB).

Distribution: Tropical Africa, India and Pakistan.

Dombeya wallichii (Lindl.) Benth. \& Hook. f., Gen. Pl. 1: 221 (1867); Bailey, Stand. Cycl. Hort. 1: 1066 (1947); Menninger, Fl. Tr. World : 268 (1962).

A large evergreen shrub or small tree. Leaves large, cordate with acute to acuminate apex and cordate base. Flowers bright pink or red, fragrant. Stigma exserted. Fruit a capsule, ovate-oblong, densely villous. Planted in the gardens. Flowering and fruiting: April-July.

Specimens examined: Dhaka: s. loc., 10.2.1947, Sukdeo, s.n. (DUSH); Dr. Kanago’s garden, 10.2.1947, Sukdeo, s.n. (DUSH); C \& B Nursery, 18.2.1966, S.K. Dutta 111 (DUSH); Dhaka, January 1942, A.K. Acharja, s.n. (DUSH).

Distribution: Indigenous to Madagascar, introduced elsewhere.

FIRMIANA Marsigli in Saggi, Sci. Acad. Padova 1: 114, 116 (1786); Kostermans, Reinwandtia 4: 281 (1957).

Type: Sterculia platanifolia L. f. [Firmiana simplex (L.) Wight]

Firmiana colorata (Roxb.) R. Br. in Bennet \& R. Br., P1. Jav. Rar.: 235 (1844); Kosterm., Reinwardtia 4: 285 (1957); Abedin and Ghafoor in Nasir \& Ali (eds), Fl. W. Pak. 99: 22 (1976); Malick in Sharma \& Sanjappa (eds), Fl. India 3: 420 (1993); Verdcourt in Dassanayake et al., Rev. Handb. Fl. Ceyl. 9: 437 (1995). Sterculia colorata Roxb., P1. Corom. 1: 26, t. 25 (1795); Fl. Ind. ed. Carey 3: 146 (1832); Mast. in Hook. f., Fl. Brit. Ind. 1: 359 (1874); Prain, Beng. P1. 1: 274 (1903). Erythropsis colorata (Roxb.) Burk., Gard. Bull. Singapore 5: 231 (1931). Firmiana rubriflora Kosterm., Reinwardtia 6: 293 (1962).

A medium-sized spreading, deciduous tree with fluted stem. Leaves crowded at the end of branchlets, palmately 3-5 lobed, lobes triangular, with acute to cuspidate apex and cordate to truncate base. Flowers scarlet or orange-red, polygamous. Fruit a follicle, oblong. Flowering and fruiting: March-June.

Specimens examined: Chittagong: Jaldi, Napura, 3.4.1921, J.M. Cowan 1281 (E); Sitakund, 10.1.1851, J.D. Hooker \& T. Thomson (K). Chittagong Hill Tracts: Raimatang hill, 6.4.1947, 
S.M. Sircar, s.n. (DUSH). Cox’s Bazar: Moheshkhali, Garakghata Kalmadia, 7.3.1978, Khan et al. K 4854 (DACB). Sylhet: s. loc. \& dies, Wallich no. 1119 (K). Tangail: On the way to Madhupur, 13.4.1997, A.M. Huq s.n. (DACB).

Distribution: Bhutan, China, India, Indonesia, Malaysia, Myanmar, Nepal, Pakistan, Sri Lanka, Thailand and Vietnam.

GUAZUMA Mill., Gard. Dict. Abridg. ed. 4: 2 (1754); Benth. \& Hook. f., Gen. P1. : 225 (1862). Type: Guazuma ulmifolia Lam.

Guazuma ulmifolia Lam., Encycl. Math. Bot. 3: 52 (1789); Robyns in Ann. Miss. Bot. Gard. 51: 102, f. 7 (1964); Abedin and Ghafoor in Nasir \& Ali (eds), Fl. W. Pak. 99: 10 (1976); Malick in Sharma \& Sanjappa (eds), Fl. India 3: 424 (1993); Verdcourt in Dassanayake et al., Rev. Handb. Fl. Ceyl. 9: 421 (1995). Theobroma guazuma L., Sp. P1.: 782 (1753). Guazuma tomentosa Kunth in H.B.K., Nov. Gen. Sp. 5: 32 (1823); Mast. in Hook. f., Fl. Brit. Ind. 1: 375 (1874); Prain, Beng. P1. 1: 278 (1903).

A small to moderate sized tree. Young twigs covered with rusty-brown or light grey stellate hairs. Leaves oblong-lanceolate with acuminate apex and obliquely cordate base. Flowers yellow. Fruit a capsule, oblong, covered with black tubercles. Flowering and fruiting: January-September.

Specimens examined: Dhaka: Govt. nursery, 30.4.1945, S.K. Sen, s.n. (DUSH). Jessore: s. loc., 23.2.1969, L. Nessa 28 (DUSH). Noakhali: 30.10.1873, C.B. Clarke 19931 (BM).

Distribution: A native of tropical America and Java, cultivated in India, Myanmar and Pakistan.

HELICTERES L., Sp. P1.: 963 (1753); Gen. P1. ed. 5: 411 (1754); Benth. \& Hook. f., Gen. P1.: 220 (1862).

Type Helicteres isora L.

\section{Key to the species:}

1. Ripe carpels spirally twisted.

H. isora

- $\quad$ Ripe carpels straight, not twisted.

2. Leaves with obtuse or slightly oblique base; petals pale violet; stamens 10; ovary hairy.

H. elongata

- $\quad$ Leaves with auriculate or cordate base; petals white or yellow; stamens 15; ovary glabrous.

H. viscida

Helicteres elongata Wall. ex Boj. in Hort. Maurit.: 35 (1837); Mast. in Hook. f., Fl. Brit. Ind. 1: 365 (1874); Kurz, Fl. Burm. 1: 144 (1877); Craib, Fl. Siam. Enum. 1: 171 (1925); Malick in Sharma \& Sanjappa (eds), Fl. India 3: 425 (1993). Helicteres plebeja Kurz, J. Asiat. Soc. Beng. 39(2): 67 (1870); Mast. in Hook. f., Fl. Brit. Ind. 1: 366 (1874); Craib, Fl. Siam. Enum. 1: 174 (1925). Helicteres glabriuscula Wall. ex Mast. in Fl. Brit. Ind. 1: 366 (1874).

A diffuse straggling shrub with slender branchlets and stellate hairs on all parts. Leaves oblong-lanceolate to obliquely ovate, with acute to acuminate apex and obtuse or slightly oblique base. Flowers pale violet. Fruit a follicle, cylindrical, hairy, beaked. Flowering and fruiting: JuneDecember.

Distribution: China, India, Myanmar, Thailand and Vietnam. 
Note: Malick (1993) reported the occurrence of this species from Bangladesh without citing any specific locality. Although Ahmed et al. (2009) cited this species from Bangladesh, however no specimen belonging to this species was available for citation.

Helicteres isora L., Sp. P1. : 963 (1753); Roxb., Fl. Ind. ed. Carey 3: 143 (1832); Wight, Icon. Pl. Ind. Dr. : t. 180 (1839); Mast. in Hook. f., Fl. Brit. Ind. 1: 365 (1874); Kurz, Fl. Burm. 1: 142 (1877); Prain, Beng. P1. 1: 275 (1903); Ridl., Fl. Mal. Pen. 1: 281 (1922); Abedin and Ghafoor in Nasir \& Ali (eds), Fl. W. Pak. 99: 13 (1976); Nicolson et al., Intepr. Hort. Mal. : 253 (1988); Malick in Sharma \& Sanjappa (eds), Fl. India 3: 426 (1993); Verdcourt in Dassanayake et al., Rev. Handb. Fl. Ceyl. 9: 426 (1995).

A large shrub or small tree, stellately hairy throughout. Leaves ovate, broadly elliptic or elliptic-obovate with cuspidate apex and cordate or rounded base. Flowers orange-red with black dots on inner part of the corolla. Fruit a follicle, cylindrical, spirally twisted with an apical beak. Flowering and fruiting: April-December.

Specimens examined: Dhaka: Savar, 10.9.1868, C.B. Clarke 7665 (K); Balda garden, 21.6.1966, S.K. Dutta 148 (DUSH); Savar, 7.8.1969, L. Nessa 110 (DUSH); Savar, near cantonment, 18.8.1977, Khan \& Huq K 4513 (DACB); Near Nayerhat, June 1982, Yusuf s. $n$. (DACB); Dhaka University Botanic garden, 8.10.2011, M.Z. Uddin, s.n. (DUSH). Chittagong: Banskhali, Jaldi beat, 14.5.1990, Khan et al. K 8351 (DACB). Tangail: Gorai, 31.3.1973, Khan \& Huq K 2975 (DACB); Gorai, 19.8.1973, A.M. Huq 1061 (DACB).

Distribution: Australia, Cambodia, China, India, Indonesia, Malaysia, Nepal, Pakistan, Sri Lanka, Thailand and Vietnam.

Helicteres viscida Bl., Bijdr. 1: 79 (1825); Kurz, Fl. Burm. 1: 143 (1877); Ridl., Fl. Mal. Pen. 1: 281 (1922); Craib, Fl. Siam. Enum. 1: 175 (1925). Helicteres spicata Colebr. ex Mast. var. lamigera Mast. in Hook. f., Fl. Brit. Ind. 1: 366 (1874). Helicteres pulchella Wall. ex Boj. in Hort. Maurit.: 35 (1837).

A shrub with stellate hairs on all parts. Leaves ovate-oblong to lanceolate with cuspidate apex and auriculate or cordate base. Flowers white or yellow. Fruit a capsule, oblong or cylindrical, beaked, covered with shaggy hairs. Flowering and fruiting: July-March.

Specimen examined: Chittagong: Jaldi Range, Boilchari, 2.12.1920. J.M. Cowan 1618 (E).

Distribution: China, Indonesia, Laos, Malaysia, Myanmar, Thailand and Vietnam.

HERITIERA Ait., Hort. Kew. 3: 546 (1789); Benth. \& Hook. f., Gen. P1. : 219 (1862).

Balanopteris Gaertn., Fruct. 2: 94, tt. 98, 99 (1791).

Type: Heritiera littoralis Ait.

\section{Key to species:}

1. Samara with a longitudinal ridge; leaves with faint secondary

nerves and veins.

- $\quad$ Samara winged or wing-like; leaves with prominent secondary nerves and veins.

2. Fruit body up to $2 \times 1 \mathrm{~cm}$; epicarp dull; pistil and stamens on a

H. fomes slender column with parallel sides.

- $\quad$ Fruit body not less than $5 \times 3 \mathrm{~cm}$; epicarp shining; pistil and stamens on a based column, narrowed in the distal half.

H. littoralis 

with 8-11 pairs of lateral nerves.

- $\quad$ Anthers in regular rings on androgynophores; leaves with 4-5 pairs of lateral nerves.

Heritiera fomes Buch.- Ham. in Symes, Embassy Ava ed. 2, 3: 319, t. 28 (1800); Mast. in Hook. f., Fl. Brit. Ind. 1: 363 (1874); Kosterm., Reinwardtia 4: 490 (1959); Malick in Sharma \& Sanjappa (eds), Fl. India 3: 428 (1993). Heritiera minor Roxb., Hort. Beng.: 50 (1814), Fl. Ind. ed. Carey 3: 142 (1832) non Lam. (1797); Prain, Beng. P1. 1: 274 (1903).

A moderate-sized evergreen tree. Leaves spirally arranged, elliptic-lanceolate or ovate, with acute to mucronate apex and tapering to rounded base. Male flowers with 5-10 stamens. Female flowers with 4-6 carpels; epicarp dull. Fruits ellipsoid or globular, woody. Flowering and fruiting: September-December.

Specimens examined: Barguna: Patharhat, 20.3.1989, Huq et al. H 9187 (DACB). Chittagong: Matamori Reserve, Chokaria, 1.4.1920, J.M. Cowan 780 (E); Charandrin, 15.4.1927, J.M Cowan s.n. (E); Garjania, s. dies, J.M. Cowan 456 (E). Chittagong Hill Tracts: Matangi reserve, 7.4.1920, J.M. Cowan 780 (E). Cox's Bazar: Baghkhali river, Kurish Kool, 4.12.1944, J. Sinclair 3836 (E); Chakaria Sundarban, near Dulahazara, 12.6.1979, Khan, Huq \& Rahman, K 5595 (DACB). Khulna: Dec. 1888, C.B. Clarke 24893 (K); Sundarbans, Oct. 1881, J.S. Gamble 10097 (E); Sundarbans, July 1890, R. Quinnell s. n. (E); Jassore, 14.6.1874, C.B. Clarke 21769 (BM); Sundarbans, 31.1.1895, Heinig s. n. (BM); Sundarban tidal forest, 20.6.1966, M. Ismail \& M. Bhowmik 180 (DUSH); Sundarban, Bhadra, 23.3.1970, M.S. Khan 1919 (DUSH); Sundarban, Kotka, 22.1.1995, M.A. Hassan 1224 (DUSH); Sundarban, Jongra, 5.11.2001, N. Uddin \& F. Deodatus N 1151 (DACB);. Satkhira: Munshiganj, Burigualini, 11.12.1989, Huq et al. H 9368 (DACB).

Distribution: India, Myanmar and Thailand.

Heritiera littoralis Dryand. in Ait., Hort. Kew. ed. 1, 3: 546 (1789); Mast. in Hook. f., Fl. Brit. Ind. 1: 363 (1874); Kurz, Fl. Burm. 1: 140 (1877); Ridl., Fl. Mal. Pen. 1: 279 (1922); Craib, Fl. Siam. Enum. 1: 170 (1925); Kosterm., Reinwardtia 4: 490 (1959).

A small to medium-sized evergreen tree. Leaves ovate to oblong, with acute apex and obtuse to subtruncate base. Male flowers with 8-10 stamens. Female flowers with 4-5 carpels. Fruit a samara, ellipsoid, woody with a longitudinal ridge, epicarp shining. Flowering and fruiting: MayDecember.

Specimen examined: s. loc. \& dies, P. Maheshwary, 13.9.49, s.n. (DUSH).

Distribution: Australia, Cambodia, China, India, Indonesia, Malaysia, Myanmar, the Philippines, Sri Lanka, Thailand, Vietnam and East Africa.

Heritiera macrophylla Wall. ex Kurz in J. Asiat. Soc. Beng. 42(2): 61 (1873); Kurz, Fl. Burm. 1: 141 (1877); Kanjilel et al., Fl. Assam 1: 155 (1934); Kosterm., Reinwardtia 4: 502 (1959); Malick in Sharma \& Sanjappa (eds), Fl. India 3: 430 (1993).

A medium-sized tree. Leaves oblong, with acute apex and obtuse to slightly cordate base. Male flowers with 6-8 stamens; anthers irregularly clumped on androgynophore. Female flowers with 1 carpel. Fruit a samara, ellipsoid, with one apical fish-tail wing. Flowering and fruiting: November-February. 
Specimens examined: Khulna: Sundarbans, 13.9.49, P. Maheswary, s.n. (DUSH); Sundarbans, 22¹0’ N 8950’E, Apr. 1984, J.M. Sandom 15 (K); Sundarbans, 22¹0’N 89 35’ E, June 1984, F.R. Miller 33 (K); Sundarbans, 22²5’ N, 89²5’ E, Apr. 1984, J.H. Sandom 36 (K).

Distribution: Cambodia, China, India, Laos, Myanmar, Thailand and Vietnam.

Heritiera papilio Bedd., Fl. Sylhet : t. 218 (1872); Mast. in Hook. f., Fl. Brit. Ind. 1: 363 (1874); Malick in Sharma \& Sanjappa (eds), Fl. India 3: 430 (1993). Heritiera acuminata All. ex Kurz in J. Bot. 12: 65, t. 141, f. 1, 1-3 (1874); Kanjilal et al., Fl. Assam 1: 155 (1934).

A small to moderate-sized tree. Leaves variable in shape, lanceolate, oblanceolate or oblong to elliptic with obtuse base. Male flowers with slender staminal column; anthers in regular rings on androgynophore. Female flowers with 5-6 ovary. Fruit a single-seeded samara. Flowering and fruiting: April-December.

Specimen examined: Sylhet: Wallich Cat. no. $7836(\mathrm{~K})$.

Distribution: India.

KLEINHOVIA L., Sp. Pl. ed. 2: 1365 (1763); Benth. \& Hook. f., Gen. Pl. 1: 219 (1867).

Kleinhovia hospita L., Sp. Pl. : 1365 (1763); Mast. in Hook. f., Fl. Brit. Ind. 1: 364 (1874); Gagnep. in Fl. Gen. I.-C. 1: 497 (1911); Ridl., Fl. Mal. Pen. 1: 280 (1922); Craib, Fl. Siam. Enum. 1: 171 (1925); Kou-mei, Fl. Reipubl. Popularis Sin. 49(2): 144, t. 40 (1984).

A small tree, with straight trunk. Leaves cordate to ovate, with acute, cuspidate or rounded apex and cordate to truncate base. Flowers pink. Stamens 10, staminodes 5. Ovary 5-locular. Fruit a capsule, inflated, membranous. Flowering and fruiting: February-April.

Specimens examined: Dhaka: s. loc., 26.11.1945, Sukdeo, s.n. (DUSH); Sadarghat, 26.12.1943, Atul, s.n. (DUSH); Sadarghat, 26.12.1943, S.K. Sen, s.n. (DUSH).

Distribution: Australia, China, India, Indonesia, Malay Peninsula, Philippines, Polynesia, Sri Lanka, Thailand and tropical Africa.

MELOCHIA L., Sp. P1. : 674 (1753); Gen. P1. ed. 5: 304 (1754); Benth. \& Hook. f., Gen. P1. : 223 (1862).

Lectotype: Melochia corchorifolia L.

Melochia corchorifolia L., Sp. P1. : 675 (1753); Mast. in Hook. f., Fl. Brit. Ind. 1: 374 (1874); Prain, Beng. P1. 1: 277 (1903); Malick in Sharma \& Sanjappa (eds), Fl. India 3: 441 (1993); Verdcourt in Dassanayake et al., Rev. Handb. Fl. Ceyl. 9: 414 (1995).

Annual herb, young parts sparsely hairy. Leaves ovate, ovate-lanceolate, oblong-ovate or suborbicular with truncate or obtuse base and acute apex. Flowers pinkish. Fruit a globose or subglobose capsule. Flowering and fruiting: March-June.

Specimens examined: Chittagong: Ganjania, s. dies., J.M. Cowan 716 (E); Sitakund 6.1.1851, J.D. Hooker 408 (K). Cox's Bazar: Korantan Quarter Municipal, roadside of a small tank, 17.8.1943, J. Sinclair 3132 (E); St. Martin’s Island, 29.10.1963, M.S. Khan 654 (DUSH); Teknaf range, 5.10.1991, Khan et al. K 8596 (DACB). Dhaka: Savar, Jahangirnagar University campus, 31.10.1999, Rezia Khatun 2010 (DACB). East Bengal: s. loc., 4.6.1850, J.D. Hooker s.n. (K). Rajshahi: Near Godagari, 17.11.1988, Huq et al. H 8670 (DACB). Noakhali: Maijdee, 1.11.63, Din Mohammad 34 (DUSH). Sylhet: s. loc. 18.9. 1850, J.D. Hooker s.n. (K).

Distribution: Australia, China, India, Indonesia, Malay Peninsula, Myanmar, Philippines, Polynesia, Thailand and Vietnam. 
PENTAPETES L., Sp. P1. : 698 (1753); Benth. \& Hook. f., Gen. P1. 1: 222 (1867). Moranda Scop., Introd.: 289 (1777).

Type. Pentapetes phoenicea L.

Pentapetes phoenicea L., Sp. Pl. 2: 698 (1762); Wight \& Arn., Prodr. 1: 67 (1834); Mast. in Hook. f., Fl. Brit. Ind. 1: 371 (1874); Prain, Beng. P1. 1: 277 (1903); Ridl., Fl. Mal. Pen. 1: 284 (1922); Craib, Fl. Siam. Enum. 1: 178 (1925); Malick in Sharma \& Sanjappa (eds), Fl. India 3: 443 (1993); Verdcourt in Dassanayake et al., Rev. Handb. Fl. Ceyl. 9: 412 (1995). Pentapetes angustifolia Bl., Bijdr.: 87 (1825).

Annual herb to undershrub with few scattered stellate hairs on the bark. Leaves linear lanceolate, with acuminate or cuspidate apex and cuneate or obtuse base. Flowers pink to red. Fruit a globose or subglobose capsule, 5-valved. Flowering and fruiting: September-January.

Specimens examined: Chittagong: Matamori Reserve Range, 2.9.1920, J.M. Cowan 811 (E); 13.9.1920, J.M. Cowan 866 (E). Dhaka: s. loc., 10.9.1818, C.B. Clarke 7703 (BM). Narayanganj: Fatullah, 19.4.1973, A.M. Huq 1088 (DACB). Rajshahi: Rajshahi University campus, 11.11.1985, Khan \& Mia K 7341 (DACB). Rangamati: Pablakhali, 30.4.1977, Huq \& Rahman H 3281 (DACB); Pablakhali, 26.9.1977, Khan et al. K 4682 (DACB).

Distribution: Australia, China, India, Indonesia, Japan, Malaysia, Myanmar, Nepal, Philippines, Sri Lanka, Thailand and Vietnam.

PTEROSPERMUM Schreb., Gen. 2: 461 (1791); Benth. \& Hook. f., Gen. P1. 1: 220 (1867). Velaga Adans., Fam. 2: 398 (1763). Sczegleewia Turcz., Bull. Soc. Nat. Mosc. 31(1): 233 (1858).

\section{Key to the species:}

1. Capsules angular; leaves peltate; petals glabrous on both

P. acerifolium surfaces; style glabrous.

- $\quad$ Capsules not angular; leaves not peltate; petals hairy on outer surface; styles hairy at least on lower half.

2. Leaves with a slightly oblique base, sparsely hairy or P. lanceaefolium glabrescent on lower surface; fruiting calyx caducous.

- $\quad$ Leaves with strongly sagittate base, woolly on lower surface; fruiting calyx persistent.

Pterospermum acerifolium (L.) Willd., Sp. P1. 3: 729 (1800); Mast. in Hook. f., Fl. Brit. Ind. 1: 368 (1874); Kurz, Fl. Burm. 1: 145 (1877); Prain, Beng. P1. 1: 276 (1903); Brandis, Ind. Trees : 91 (1906); Craib, Fl. Siam. Enum. 1: 175 (1925); Abedin and Ghafoor in Nasir \& Ali (eds), Fl. W. Pak. 99: 12 (1976); Malick in Sharma \& Sanjappa (eds), Fl. India 3: 448 (1993). Pentapetes acerifolia L., Sp. Pl.: 698 (1753).

A medium-sized to large evergreen tree. Leaves polymorphous, peltate, with cordate base. Flowers white or yellowish, fragrant. Fruit a capsule, oblong, woody, rusty brown, glabrescent. Flowering and fruiting: February-June.

Specimens examined: Chittagong: Cheval Reserve forest, 27.2.1968, Majumdar \& Islam 39A, 39B (K); Sitakund, 13.1.1851, J.D. Hooker \& T. Thomson 621 (K). Dhaka: s. loc., March 1938, N.K. Chatterji, s.n. (DUSH); Ramna, 10.2.1954, Shahjahan, s.n. (DUSH); Balda garden, 17.2.1988, Rezia, Huq \& Mia R 231 (DACB). Habiganj: Chunarughat, Kalenga beat, 1.4.1997, 
A.M. Huq 10410 (DACB). Khulna: Near railway station, 16.6.1982, A.M. Huq 5537 (DACB). Sylhet: Wallich no. 11702 (K). Munshiganj: Vikrampur, 30.10.1868, C.B. Clarke 7937 (BM).

Distribution: Bhutan, China, India, Laos, Malaysia, Myanmar, Nepal, Pakistan and Thailand.

Pterospermum lanceaefolium Roxb., Fl. Ind. ed. 2, 3: 163 (1832); Mast. in Hook. f., Fl. Brit. Ind. 1: 368 (1874); Malick in Sharma \& Sanjappa (eds), Fl. India 3: 450 (1993). Pterospermum jackianum Wall. ex Mast. in Hook. f., Fl. Brit. Ind. 1: 367 (1874); Ridl., Fl. Mal. Pen. 1: 283 (1922); Craib, Fl. Siam. Enum. 1: 176 (1925). Pterospermum pierrei Hance. J. Bot. 15: 329 (1877); Craib, Fl. Siam. Enum. 1: 177 (1925). Pterospermum insulare Pierre, Fl. For. Cochinch.: t. 180 (1888).

A small to medium-sized tree, young parts tomentose. Leaves lanceolate-oblong, oblanceolate or obovate-oblong, with acute, acuminate or cuspidate apex and slightly oblique base. Flowers yellowish or white, fragrant. Fruit an elliptic to ovoid capsule, densely hairy. Flowering and fruiting: October-April.

Specimens examined: Sylhet: Wailich no. 1172 (K, BM); Sylhet station, 24.9.1872, C.B. Clarke 17955 (BM); Jafflong (37 miles away), 30.4.1981, Huq, Rahman \& Mia H 5121 (DACB); Jafflong, 20.10.1986, Huq \& Mia H 7904 (DACB).

Distribution: China, India, Malaysia, Myanmar, Thailand and Vietnam.

Pterospermum semisagittatum Buch.-Ham. ex Roxb., Fl. Ind. ed. Carey 3: 160 (1832); Mast. in Hook. f., Fl. Brit. Ind. 1: 368 (1874); Kurz, Fl. Burm. 1: 146 (1877); Prain, Beng. P1. 1: 276 (1903); Craib, Fl. Siam. Enum. 1: 177 (1925); Malick in Sharma \& Sanjappa (eds), Fl. India 3: 453 (1993).

A small to moderate-sized tree. Leaves oblong to oblong-lanceolate, with acute to cuspidate apex and sagittate base. Flowers white, fragrant. Fruit a capsule, cylindrical or elliptical, rustytomentose. Flowering and fruiting: April-August.

Specimens examined: Chittagong: Jaldi Range, Taballa Chera, 6.5.1921, J.M. Cowan 399 (E); Razu Reserved Range, 17.5.1920, J.M. Cowan s. n. (E); Gobania, 31.5.1920, J.M. Cowan 335 (E); Rezu Range, 16.8.1920, J.M. Cowan 215 (E); Mirsari, 19.5.1920, Cowan 126 (E); s. loc., Nov. 1862, Pierre 9718 (E, BM); Sitakund 5.1.1851, J.D. Hooker \& T. Thomson 374 (K); s.loc., October 1940, S.K. Sen, s.n. (DUSH); Cheval Reserve forest, 10.6.1967, S. Majumdar \& A. Islam 73A (K); 24.7.1966, S. Mazumdar \& A. Islam 73B (K); Harbang to Aziznagar, 13.6.1979, Khan, Huq \& Rahman K 5609 (DACB); Chunati Game Reserve, 22.4.1994, Huq \& Mia H 10318 (DACB). Chittagong Hill Tracts: Phasoha, 28.2.1879, J.S. Gamble 6725A (K); Sept. 1886, Dr. King's Coll. 232 (K, BM). Cox's Bazar: Kelatali, 21.3.1945, J. Sinclair 4054 (E); Harikhola, Whykong range, 2.6.2000, Khan et al. K 10523 (DACB). Sylhet: Tamabil, 29.4.1981, Huq, Rahman \& Mia H 5078 (DACB).

Distribution: Cambodia, India, Laos, Myanmar, Sri Lanka and Thailand.

PTERYGOTA Schott \& Endl., Melet. Bot. : 32 (1832); Hutch., Gen. Fl. P1. 2: 520 (1967).

Type: Sterculia alata Roxb. [Pterygota alata (Roxb.) R. Br.)].

Pterygota alata (Roxb.) R. Br. in Benn., Pl. Jav. Rar. : 234 (1834); Kochumenn in Whitmore, Tr. Fl. Malaya 2: 371 (1973); Abedin and Ghafoor in Nasir \& Ali (eds), Fl. W. Pak. 99: 23 (1976); Malick in Sharma \& Sanjappa (eds), Fl. India 3: 455 (1993). Sterculia alata Roxb., P1. Corom. 3: 84, t. 287 (1819); Fl. Ind. ed. Carey 3: 152 (1832); Mast. in Hook. f., Fl. Brit. Ind. 1: 360 (1874); Kurz, Fl. Burm. 1: 134 (1877); Prain, Beng. P1. 1: 274 (1903). Erythropsis roxburghii Schott \& Endl., Melet. Bot.: 33 (1832). Sterculia teynii Bedd., Fl. Sylv. : t. 230 (1872). 
A large deciduous tree with narrow conical crown. Leaves broadly ovate with acute to broadly acute apex and cordate or truncate base, usually clustered at the end of brachlets. Flowers brownish-yellow. Fruit a woody follicle, globose to ellipsoid, pubescent. Flowering and fruiting: December-May.

Specimens examined: Chittagong: Chunati Game Reserve, 24.4.1994, Huq \& Mia H. 10332 (DACB). Chittagong Hill Tracts: s. loc., Mar. 1880, J.S. Gamble 7898 (K). Dhaka: s. loc., 24.2.1947, Sukdeo, s.n. (DUSH); Azimpur Army Recruiting office compound, 1.11.1963, A.F. Muhammad 34 (DUSH); Ramna, 3.2.1947, S.K. Sen, s.n. (DUSH); Dhaka University TSC area, 10.9.1982, A.M. Huq 5688 (DACB). Sylhet: Wallich no. 1122, 1 (K).

Distribution: Bhutan, China, India, Malaysia, Myanmar, Pakistan, Philippines, Thailand and Vietnam.

SCAPHIUM Schott. \& Endl., Melet. Bot. : 33 (1832).

Type: Scaphium wallichii Schott. \& Endl.

Scaphium scaphigerum (Wall. ex G. Don) Guib. \& Planch., Hist. Nat. Dro. Sim. ed. 7, 3: 632 (1876); Kosterm., J. Sci. Res. Indo. 2(1): 3 (1953). Sterculia scaphigera Wall., Cat. no. 1130 (1828); Mast. in Hook. f., Fl. Brit. Ind. 1: 361 (1874). Scaphium wallichii Schott \& Endl., Melet. Bot. : 33 (1832); Benn. \& R. Br., Pl. Jav. Rar. : 236 (1844).

A medium-sized to large deciduous tree. Leaves ovate to ovate-oblong, with acute to acuminate apex and obtuse to truncate base. Petals absent. Male flowers with 10-15 stamens; female flowers with 5 carpels. Fruit a follicle, boat-shaped, membraneous. Flowering and fruiting: February-May.

Specimens examined: Rangamati: 50 miles south of Rangamati, Apr. 1934, Macalpine \& W.J.L. Wenger 476 (K); May 1933, W.J.L. Wenger s. n. (K).

Distribution: Cambodia, Malay Peninsula, Myanmar and Thailand.

STERCULIA L., Sp. P1. : 1007 (1753); Benth. \& Hook. f., Gen. P1. 1: 217 (1862).

\section{Type: Sterculia foetida L.}

\section{Key to species:}

1. $\quad$ Leaves digitate or palmately lobed. 2

- $\quad$ Leaves not lobed. $\quad 5$

2. Leaves digitate. 3

- $\quad$ Leaves palmately lobed. 4

3. Leaflets whitish pubescent beneath; calyx lobes inflexed; S. versicolor staminal column c. $2 \mathrm{~mm}$ long.

- $\quad$ Leaflets glabrous beneath when mature; calyx lobes spreading; S. foetida staminal column c. $10 \mathrm{~mm}$ long.

4. Leaves deeply 5-7 lobed; lobes 3-fid, villous beneath; flowers S. villosa large, c. $2 \mathrm{~cm}$ wide; follicles spreading.

- $\quad$ Leaves shallowly 5-lobed, entire; softly thick velvety beneath; $\quad$ S. urens flowers small, less than $8 \mathrm{~mm}$ wide; follicles radiating.

5. $\quad$ Calyx lobes broadly ovate, spreading. $\quad 6$

$\begin{array}{lll}\text { - } & \text { Calyx lobes linear or linear-lanceolete, connivent at the top. } & \mathbf{7}\end{array}$ 
6. Leaves glabrous above, rusty-tomentose beneath, cordate or subcordate at base.

- $\quad$ Leaves glabrous on both surfaces, more or less rounded at base.

\section{S. roxburghii}

7. Fruits lanceolate; staminal column 4-5 mm long.

- $\quad$ Fruits ovate; staminal column less than $4 \mathrm{~mm}$.

8. Leaves glabrous or glabrescent on both surfaces; calyx urceolate;

S. parviflora style glabrous.

- $\quad$ Leaves densely stellate hairy on both surface; calyx campanulate; style hairy.

S. balanghas

Sterculia balanghas L., Sp. Pl. : 1007 (1753); Mast. in Hook. f., Fl. Brit. Ind. 1: 358 (1874). Sterculia rubiginosa Vent., Jard. Malm. 2: t. 91 (1804); Mast. in Hook. f., Fl. Brit. Ind. 1: 358 (1874); Kurz, Fl. Burm. 1: 138 (1877); Ridl., Fl. Mal. Pen. 1: 271 (1922); Craib, Fl. Siam. Enum. 1: 169 (1925). Sterculia angustifolia Roxb., Fl. Ind. 3: 148 (1832); Kurz, Fl. Burm. 1: 138 (1877); Ridl., Fl. Mal. Pen. 1: 274 (1922); Craib, Fl. Siam. Enum. 1: 165 (1925); Raizada, Ind. For. 67: 245 (1941). Sterculia ensifolia Mast. in Hook. f., Fl. Brit. Ind. 1: 358 (1874). Sterculia balanghas L. var. angustifolia (Roxb.) Mast. in Hook. f., Fl. Brit. Ind. 1: 358 (1874).

A small to medium-sized evergreen tree. Leaves elliptic-oblong to obovate-oblong, stellate hairy on both surfaces, with blunt, acute or acuminate to caudate apex and rounded base. Flowers yellow or greenish-purple, fragrant. Petals absent. Fruits a follicle, oblong, narrowed at both ends. Flowering and fruiting: January-May.

Specimens examined: Bengal: s. loc. \& s.n., 1796 (BM); Wallich no. 1131 (BM); Wallich no. 1133 (BM); Rangamati: Kasalong Reserve, Mainimukh, 12.3.1940, T.V. Dent. s.n.(?)

Distribution: India, Indonesia, Malay Peninsula, Myanmar, Nepal, Sri Lanka and Thailand.

Sterculia foetida L., Sp. P1.: 1008 (1753); Roxb., Fl. Ind. ed. Carey 3: 154 (1832); Mast. in Hook. f., Fl. Brit. Ind. 1: 354 (1874); Kurz, Fl. Burm. 1: 135 (1877); Prain, Beng. Pl. 1: 274 (1903); Ridl., Fl. Mal. Pen. 1: 268 (1922); Craib, Fl. Siam. Enum. 1: 166 (1925); Abedin and Ghafoor in Nasir \& Ali (eds.), Fl. W. Pak. 99: 16 (1976); Malick in Sharma \& Sanjappa (eds), Fl. India 3: 459 (1993); Verdcourt in Dassanayake et al., Rev. Handb. Fl. Ceyl. 9: 431 (1995).

A medium to large deciduous tree. Leaves elliptic, elliptic-lanceolate or elliptic-oblong, crowded at the end of branchlets. Flowers dull red, purplish or yellow. Petals absent. Fruit a follicle, woody, boat-shaped. Flowering and fruiting: November-April.

Specimens examined: Cox’s Bazar: Kutubdia, Barkup, 27.12.1983, Huq, Rahman \& Mia H 6653 (DACB). Dhaka: s. loc., 24.2.1947, Sukdeo, s.n. (DUSH); Azimpur maternity hospital, 12.8.1964, A.F. Muhammed 180 (DUSH).

Distribution: Cambodia, China, India, Indonesia, Malaysia, Myanmar, Pakistan, Philippines, Sri Lanka, Thailand, Vietnam, Eastern Africa and North Australia.

Sterculia guttata Roxb. [Hort. Beng.: 50 (1814) nom. nud], Fl. Ind. ed. Carey 3: 148 (1832); Mast. in Hook. f., Fl. Brit. Ind. 1: 355 (1874); Brandis, Ind. Trees : 82 (1921); Craib, Fl. Siam. Enum. 1: 166 (1925); Malick in Sharma \& Sanjappa (eds), Fl. India 3: 462 (1993).

A moderate-sized deciduous tree. Leaves broadly ovate to ovate-oblong, with acute or abruptly shortly acuminate apex and rounded or slightly cordate base. Flowers white or pale 
yellow. Petals absent. Fruit a follicle, curved upward, tomentose outside. Flowering and fruiting: April-August.

Specimen examined: Sylhet: Wallich no. 1127 (BM?).

Distribution: India, Myanmar, Sri Lanka, Thailand and Vietnam.

Sterculia hamiltonii (O. Kuntze) Adelb., Blumea 5: 506 (1945); et in Backer \& Bakh. f., Fl. Java Aufl. 107: 23 (1944); Malick in Sharma \& Sanjappa (eds), Fl. India 3: 464 (1993). Clompanus hamiltonii O. Kuntze, Rev. Gen. P1. 1: 77 (1891). Sterculia coccinea Roxb. [Hort. Beng. : 50 (1814) nom. nud], Fl. Ind. ed. Carey 3: 151 (1832) non Jack (1822); Mast. in Hook. f., Fl. Brit. Ind. 1: 357 (1874). Sterculia indica Men., J. Ann. Arb. 33: 245 (1952).

A small tree. Leaves elliptic-lanceolate, oblanceolate or narrowly oblong, with abruptly short acuminate apex and tapering base. Flowers yellowish, fragrant. Fruit a follicle, oblong-lanceolate, crimson-red. Flowering and fruiting: April-September.

Specimens examined: Cox's Bazar: Hill side, 19.3.1945, J. Sinclair 4046 (E). Maulvi Bazar: Kamalganj, Adampur forest beat, 20.9.2011, S.N. Uddin 4745 (DACB). Sylhet: s. loc., Wallich no. 1122 (K, BM); s. loc., 12.10.1973, Khan, Huq \& Hassan, K 3233 (DACB); Jaintapur, 19.10.1986, Huq \& Mia H 7839 (DACB).

Distribution: Bhutan, India, Myanmar and Nepal.

Sterculia parviflora Roxb. ex G. Don, Gen. Hist. 1: 516 (1831); Roxb., Fl. Ind. ed. Carey 3: 147 (1832); Mast. in Hook. f., Fl. Brit. Ind. 1: 356 (1874); Ridl., Fl. Mal. Pen. 1: 271 (1922); Malick in Sharma \& Sanjappa (eds), Fl. India 3: 468 (1993). Sterculia maingayi Mast. in Hook. f., Fl. Brit. Ind. 1: 359 (1874). Sterculia holttumii Ridl., Kew Bull. 1926: 471 (1926).

A medium-sized to fairly large deciduous tree. Leaves elliptic-oblong, with acute or shortly acuminate apex and obtuse base. Flowers yellowish brown. Petals absent. Male flowers with 10 stamens; female flowers with 5 carpels. Fruit a follicle, oblong, pubescent. Flowering and fruiting: February-July.

Specimen examined: Sylhet: S. loc. \& s. dies Wallich 1121/1 (BM).

Distribution: India, Indonesia, Malay Peninsula, Myanmar, Thailand and Vietnam.

Sterculia roxburghii Wall. [Cat. 1124 (1830) nom. nud.], P1. Asiat. Rar. 3: t. 262 (1832); Mast. in Hook. f., Fl. Brit. Ind. 1: 356 (1874); Prain, Beng. P1. 1: 274 (1903); Malick in Sharma \& Sanjappa (eds.), Fl. Ind. 3: 468 (1993). Sterculia lanceifolia G. Don, Sylhet 1: 517 (1831).

A medium-sized tree, with ashy bark. Leaves oblong-elliptic or oblanceolate, with acuminate apex and rounded or subcordate base. Racemes few-flowered, with sparingly stellate pilose pedicels. Flowers red. Fruit a follicle, ellipsoid-lanceolate. Flowering and fruiting: FebruaryAugust.

Specimen examined: Chittagong: Dhopasari, 21.3.1921, J.M. Cowan2046 (E); 2.4.1920, J.M. Cowan 156 (E). Sylhet: s. dies Wallich nos. 1124, 1125 (K).

Distribution: China and India.

Sterculia urens Roxb., P1. Corom. 1: 25, t. 24 (1795); Fl. Ind. ed. Carey 3: 145 (1832); Wight \& Arn, Prodr. 1: 63 (1834); Mast. in Hook. f., Fl. Brit. Ind. 1: 355 (1874); Prain, Beng. P1. 1: 274 (1903); Malick in Sharma \& Sanjappa (eds), Fl. India 3: 470 (1993); Verdcourt in Dassanayake et al., Rev. Handb. Fl. Ceyl. 9: 432 (1995). 
A deciduous tree. Leaves crowded at the end of branchlets, palmately 3-5 lobed, with acuminate to cuspidate apex and deeply cordate base. Flowers yellow. Fruit a follicle, oblong, ellipsoid or kidney-shaped, densely rusty pubescent. Flowering and fruiting: October-February.

Specimen examined: Chittagong: s. loc. 31.12.1850, J.D. Hooker \& T. Thomson 302 (K).

Distribution: India and Sri Lanka.

Sterculia versicolor Wall., Pl. Asiat. Rar. 1: 48, t. 59 (1830).

A large tree with spreading crown. Leaves peltate, elliptic-lanceolate with acute or shortly acuminate apex and tapering base, pubescent beneath. Flowers orange-yellow or pale yellow, fragrant. Petals absent. Fruit a slightly compressed follicle. Flowering and fruiting: March-July.

Specimen examined: Cox's Bazar: Moheshkali, 7.3.1978, Khan et al. K 4895 (DACB).

Distribution: India and Myanmar.

Sterculia villosa Roxb. [Hort. Beng. : 50 (1814), nom. nud.], Fl. Ind. ed. Carey 3: 153 (1832); Mast. in Hook. f., Fl. Brit. Ind. 1: 355 (1874); Prain, Beng. P1. 1: 274 (1903); Malick in Sharma \& Sanjappa (eds), Fl. India 3: 472 (1993). Sterculia ornata Wall. ex Kurz in J. Asiat. Soc. Beng. 42(2): 228 (1873); Kurz, Fl. Burm. 1: 136 (1877); Brandis, Ind. Trees : 81 (1921); Craib, Fl. Siam. Enum. 1: 168 (1925). Sterculia armata Mast. in Hook. f., Fl. Brit. Ind. 1: 357 (1874).

A moderate-sized deciduous tree. Leaves palmately lobed, oblong with acuminate to cuspidate apex and cordate base. Flowers pinkish-yellow. Petals absent. Male flowers with 10 stamens and female flowers with 5 free carpels. Fruit a folicle, oblong, rusty pubescent. Flowering and fruiting: February-May.

Specimens examined: Bandarban: s. loc., 3.5.1977. A.M. Huq 3354 (DACB). Chittagong: Jaldi Range, Badarmara, J.M. Cowan 2275 (E). Cox's Bazar: Kalatuli chara, 17.3.1945, J. Sinclair 4040 (E). Dhaka: s. loc., 24.2.1947, Sukdeo, s.n. (DUSH); Dhaka University Botanic Garden, 23.1.1964, A.F. Muhammed 78 (DUSH). Gazipur: Salna forest, 24.1.1968, N. Begum 83 (DUSH). Jamalpur: Gajni forest, 7.5.1982, Mia et al. M 817 (DACB). Tangail: Madhupur, Pirgacha, 14.6.1989, M.K. Mia 211,E3 (?) (DACB).

Distribution: Bhutan, Cambodia, China, India, Myanmar, Nepal, Pakistan and Thailand.

WALTHERIA L., Sp. P1. : 673 (1753); Gen. P1. ed. 5: 304 (1754); Benth. \& Hook. f., Gen. P1. : 224 (1862). Astropus Spreng., Neue Entdecj. 3: 64 (1822).

Type: Waltheria indica L.

Waltheria indica L., Sp. P1.: 673 (1753); Wight \& Arn., Prodr. 1: 67 (1834); Mast. in Hook. f., Fl. Brit. Ind. 1: 374 (1874); Prain, Beng. P1. 1: 278 (1903); Ridl., Fl. Mal. Pen. 1: 285 (1922); Craib, Fl. Siam. Enum. 1: 179 (1925). Malick in Sharma \& Sanjappa (eds), Fl. India 3: 473 (1993); Verdcourt in Dassanayake et al., Rev. Handb. Fl. Ceyl. 9: 418 (1995). Waltheria americana L., Sp. P1. : 673 (1753).

A much branched erect herb or undershrub. Leaves ovate, ovate-oblong or elliptic-ovate, with acute to rounded apex and shallowly cordate to obtuse base. Flowers yellow. Fruit a capsule, ovoid, with an acute hooked apex. Flowering and fruiting: April-December.

Specimens examined: Dhaka: Dhaka farm, 12.1.1941, Sen \& Singh, s.n. (DUSH); Nakhal para, 20.7.1969, M.M.R. Bhuiyan 158 (DUSH).

Distribution: Pantropical. 


\section{Acknowledgement}

The authors thank the authorities of the herbaria K, E, BM and DACB for allowing them to study specimens and use the library facilities.

\section{References}

Ahmed, Z.U., Hassan, M.A., Begum, Z.N.T., Khondker, M., Kabir, S.M.H., Ahmad, M., Ahmed, A.T.A., Rahman, A.K.A. and Haque, E.U. (eds.). 2009. Encyclopedia of Flora and Fauna of Bangladesh, Vol. 10. Angiosperm: Dicotyledons (Ranunculaceae - Zygophyllaceae). Asiatic Society of Bangladesh, Dhaka.

Brizicky, G.K. 1966. The genera of Sterculiaceae in the southeastern United States. J. Arnold Arbor. 47: 6074.

Cronquist, A. 1981. An integrated system of classification of flowering plants. Columbia University Press, New York.

Engler, A. and Prantl, C. 1896. Pflanzenfamilien. Leipzig.

Hsiang-Hao, H. 1984. Sterculiaceae. In: Kuo-Mei, F. (ed.), Fl. Reipubl. Popularis Sin. 49(2): 112-189.

Hooker, J.D. 1874. The Flora of British India. Vol. 1. L. Reeve \& Co. Ltd., England, pp. 353-379.

Khan, M.S. (ed.) 1972-1987. Flora of Bangladesh, Fascicles 1-19. Bangladesh National Herbarium, BARC, Dhaka.

Khan, M.S. and Rahman, M.M. (eds) 1989-2002. Flora of Bangladesh. Fascicles 40-53. Bangladesh National Herbarium, Dhaka.

Malick, K.C. 1993. Sterculiaceae. In: Sharma, B.D. and Sanjappa, M. (eds), Flora of India, Vol. 3. Botanical Survey of India, Calcutta, pp. 407-473.

Mia, M.M.K., Rahman, M.O., Hassan, M.A. and Huq, A.M. 2011. Three new records of Sterculiaceae for Bangladesh. Bangladesh J. Plant Taxon. 18(2): 153-157.

Phengklai, C. 2001. Sterculiaceae. In: Santisuk, T. and Larsen, K. (eds), Flora of Thailand. Vol. 7, Part 3. The Forest Herbarium, Royal Forest Department, Bangkok, Thailand, pp. 539-654.

Prain, D. 1903. Bengal Plants. Vol. 1. (Reprint edition 1981). Bishen Singh Mahendra Pal Singh, Dehra Dun, India, pp. 271-279.

Robyns, A. and Cautrecasas, J. 1964. Flora of Panama. Part VI. Family 117. Sterculiaceae. Ann. Miss. Bot. Gard. 51: 69-107.

Verdcourt, B. 1995. Sterculiaceae. In: Dassanayake, M.D., Fosberg, F.R. and Clayton, W.D. (eds), A Revised Handbook to the Flora of Ceylon, Vol. 9. Amerind Publishing Co. Pvt. Ltd., New Delhli, pp. 418-445. 